\title{
Imaging Findings of Acute Pancreatitis Secondary to Pancreatic Tumors
}

Hyun Young Han', MD., Kyung Sook Shin ${ }^{2}$, MD., Dong Wook Kang ${ }^{3}$, MD.

Department of Radiology, Eulji University Hospital, Korea'.Department of Radiology, Chung Nam National University Hospital, Korea².

Department of Pathology, Eulji University Hospital, Korea ${ }^{3}$

Learning objectives

Evaluate the characteristic radiologic findings of the pancreatic tumors that are presented with acute pancreatitis as initial clinical manifestations.

\section{Background}

Pancreatic tumors are difficult to be early detected because of the related symptoms are often nonspecific, subtle or even absent.

If they are manifested in acute pancreatitis without history of alcohol abuse or biliary stone, we can find those tumors more easily.

\section{Imaging findings}

From 2006 to 2017, 1506 cases of acute pancreatitis by any causes are reviewed for this study. Among them alcohol pancreatitis, gallstone pancreatitis

hyperypercalcemia, hyperlipidemia, infection, trauma medication induced pancreatitis were excluded. Only 19 cases ( 13 men, 6 women) with acute pancreatitis 2ndary to pancreatic tumor without history of alcohol abuse and biliary stone are enrolled in this study. The analysis of image findings included, location of the pancreatic tumor, tumor size, pancreatic duct dilatation, parenchymal swelling adjacent to tumor, peripancreatic fluid collection, regional or distant metastatic lymphadenopathies, vascular invasion/encasement, and venous thrombosis.

Result

Types of pancreatic tumor that were presented acute pancreatitis in this retrospective study.

\begin{tabular}{|l|c|}
\hline Types of pancreatic tumor & Value \\
\hline $\begin{array}{l}\text { IPMN (intraductal papillary } \\
\text { mucinous neoplasm) }\end{array}$ & $12(61 \%)$ \\
\hline - Benign & 10 \\
\hline - Malignant & 2 \\
\hline Metastatic pancreatic tumor & $5(28 \%)$ \\
\hline - From lung cancer & 4 \\
\hline - From rectal cancer & 1 \\
\hline Mucinous cystadenoma & $1(5.5 \%)$ \\
\hline Pancreatic adenocarcinoma & $1(5.5 \%)$ \\
\hline
\end{tabular}

Analyzed image findings in acute pancreatitis due to pancreatic tumors

\begin{tabular}{|c|c|c|}
\hline \multicolumn{2}{|l|}{ Image findings } & Data \\
\hline \multicolumn{2}{|c|}{ Mean size (cm) } & $\begin{array}{c}2.4 \\
(1.1-5.8)\end{array}$ \\
\hline \multirow{2}{*}{ Distribution } & Head & $11(55 \%)$ \\
\cline { 2 - 3 } & Body & $3(17 \%)$ \\
\cline { 2 - 3 } & Tail & $5(28 \%)$ \\
\hline Localized ductal dilatation (cm) & $0.4-0.9$ \\
\hline \multirow{2}{*}{ Parenchymal swelling } & CT grade C & $10(55 \%)$ \\
\cline { 2 - 3 } & CT grade D & $8(45 \%)$ \\
\hline \multicolumn{2}{|c|}{ Venous Thrombosis } & $1(6 \%)$ \\
\hline \multicolumn{2}{|c|}{ LAP \& Vascular invasion } & $0(0)$ \\
\hline
\end{tabular}

Key image findings in acute pancreatitis secondary to pancreatic tumor

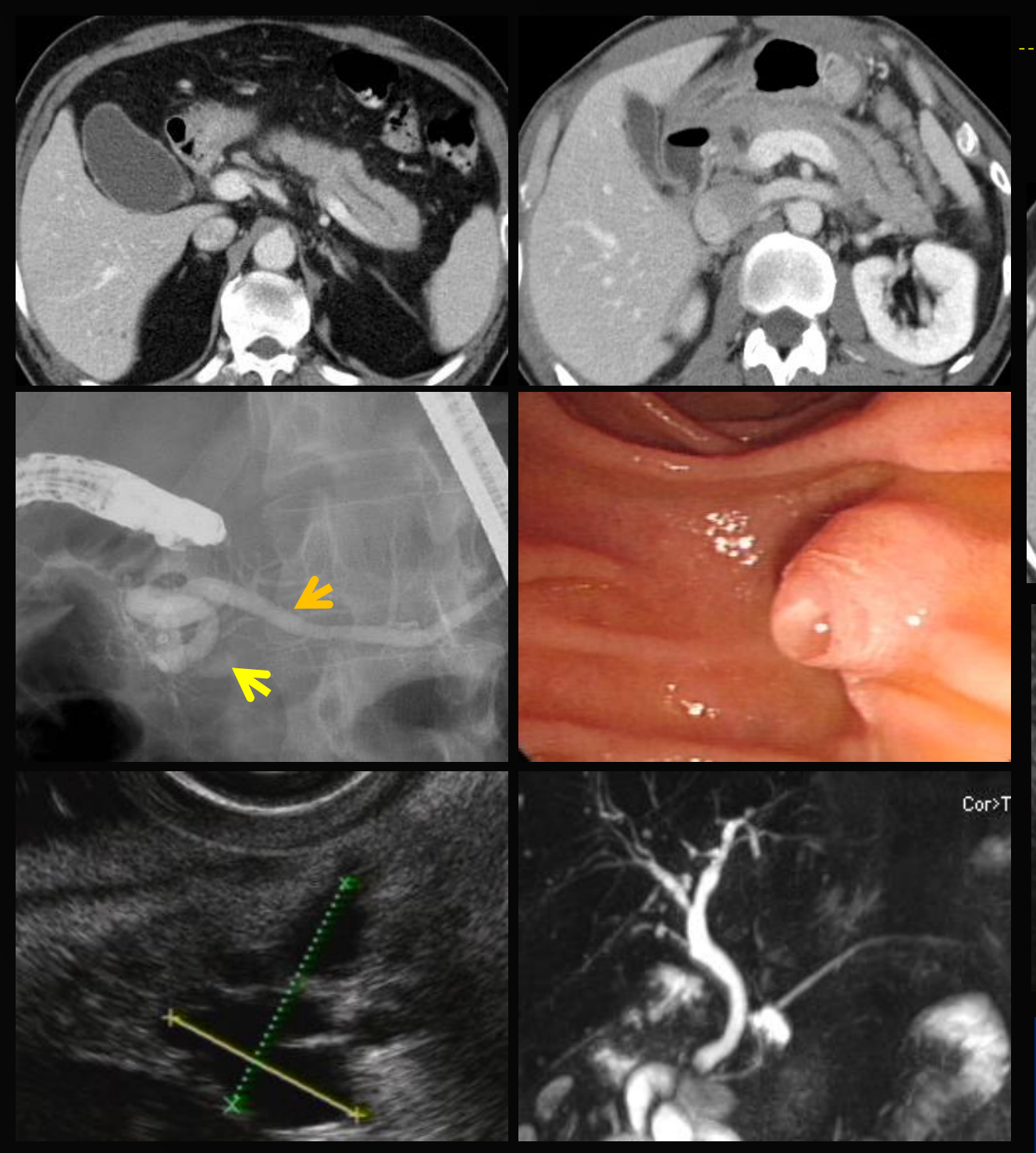

- Localized upstream duct dilatation at tumor site

Parenchymal swelling without

peripancreatic fluid collection
1) Acute pancreatitis by malignant IPMN (52/M)

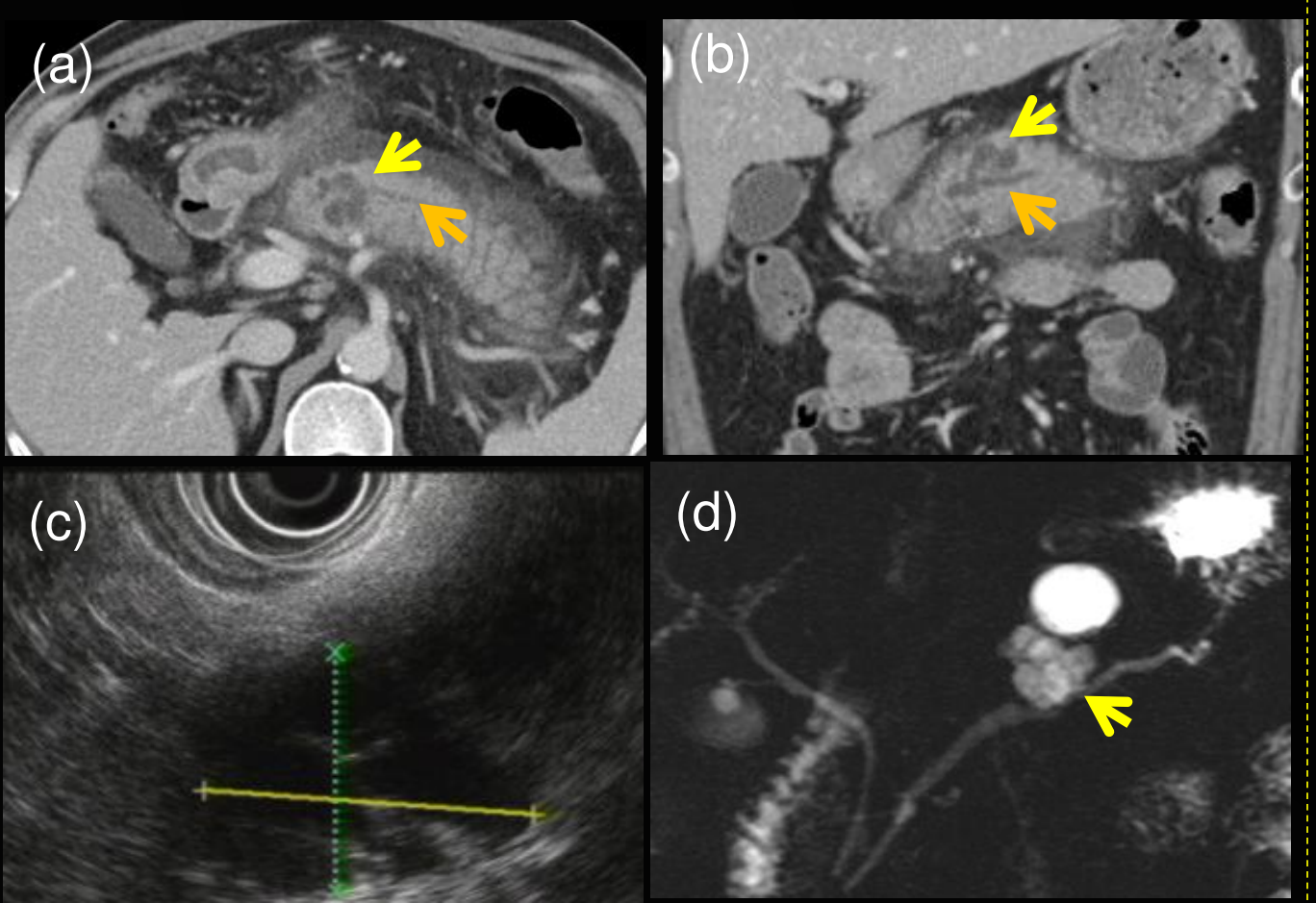

(a), (b) Abdomen CT shows diffuse pancreatic swelling, peripancreatic infiltration, fluid collection, dilated P-duct, multi-loculated cystic lesion in pancreatic body. (c), (d) EUS, MRCP depicts well defined multilocular cystic mass

2) Acute pancreatitis by malignant IPMN (76/F)

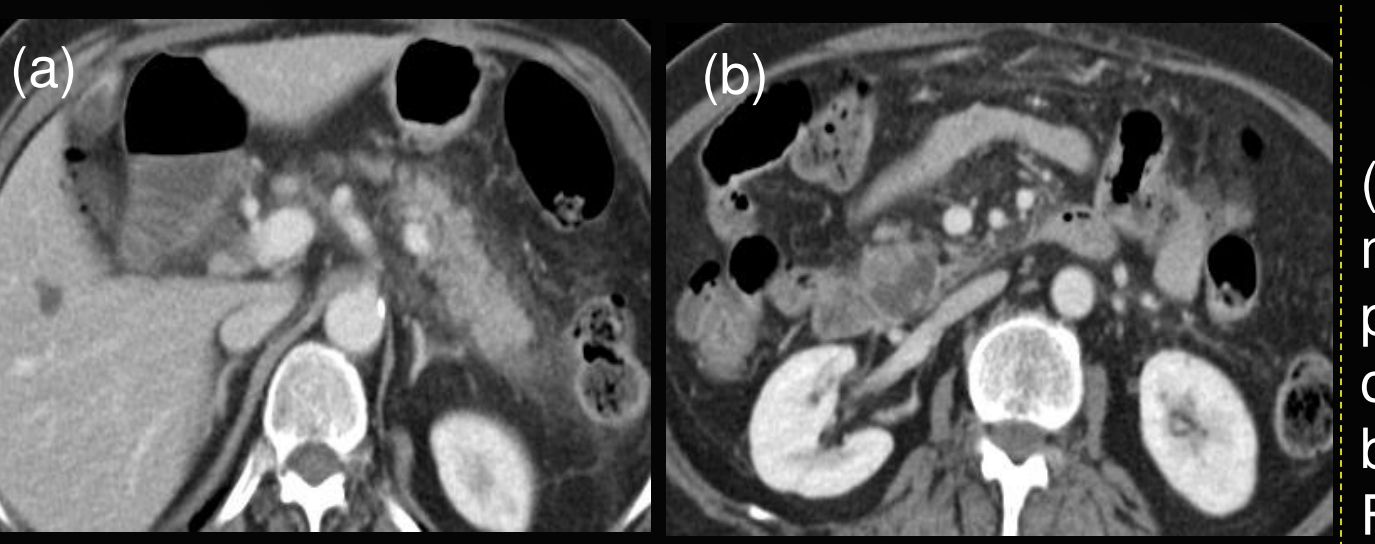

Acute pancreatitis by metastatic pancreatic cancer (57/M)
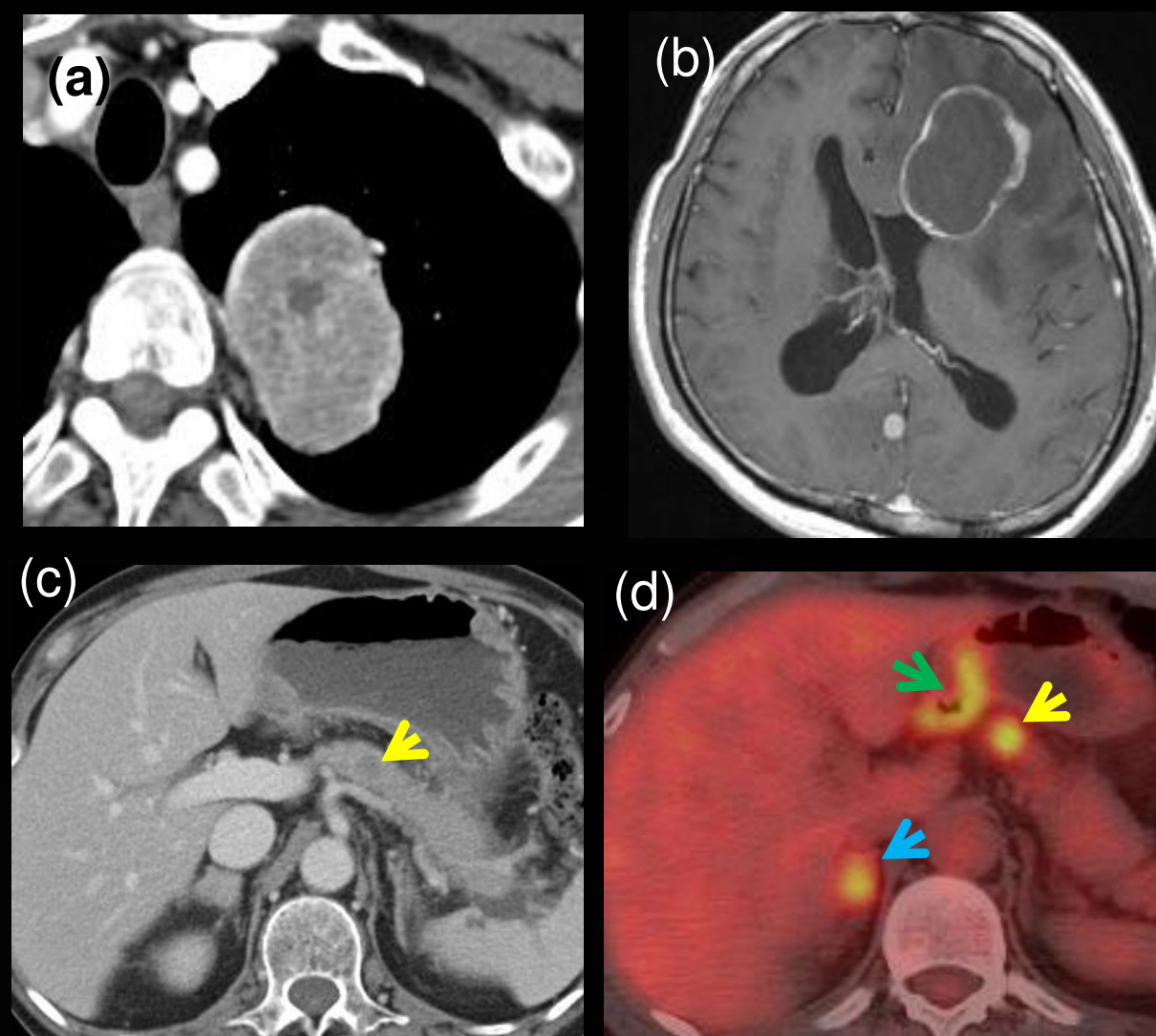

(d)

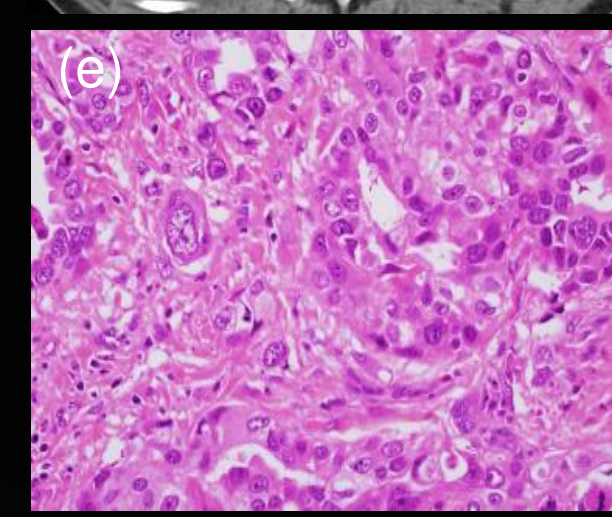

(b) Known primary lung cancer with brain metastasis. (c) Abdomen CT shows diffuse pancreatic swelling, peripancreatic fluid

collection, hypodense lesion in the pancreatic

body and right adrenal mass. (d) Multifocal hig

FDG uptake in PET/ CT indicate metastatic

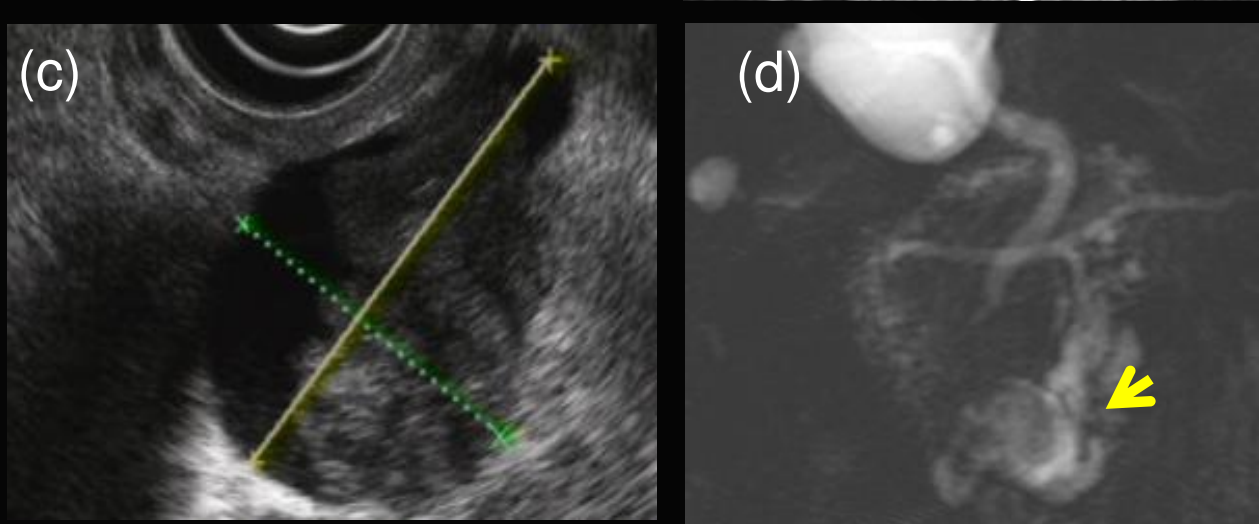

masses of pancreas \& Rt. adrenal gland.

(e) Histologic feature of metastatic carcinoma

from the lung

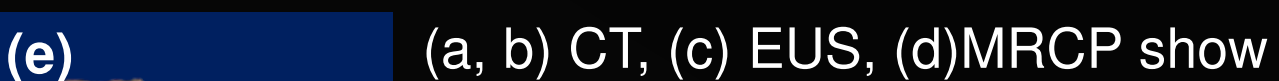
(a, b) CT, (c) EUS, (d)MRCP show
Cystic lesion with internal polypoid solid component in pancreatic head. (e) Gross features of IPMN with associated invasion

\section{Conclusion}

Acute pancreatitis w/o cholelithiasis or history of alcohol consumption can be the only one clue early detection of pancreatic tumor. IPMN is

most common tumor that can manifest acute pancreatitis in this study. Characteristic image findings in acute pancreatitis secondary to pancreatic tumor are accompanied upstream duct dilatation at tumor site \& pancreatic swelling without peripancreatic fluid collection. 